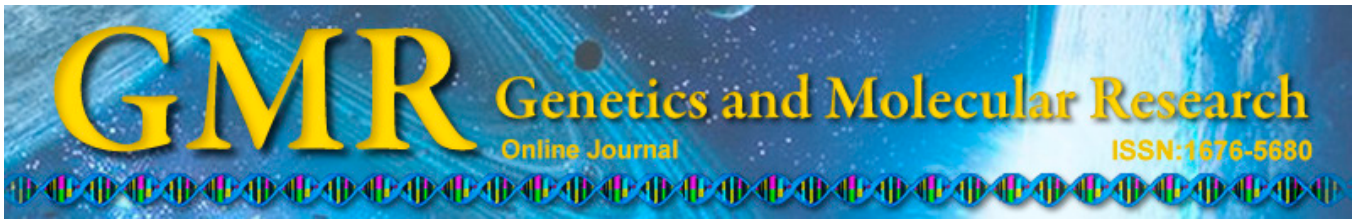

\title{
A novel mutation links to von Hippel-Lindau syndrome in a Chinese family with hemangioblastoma
}

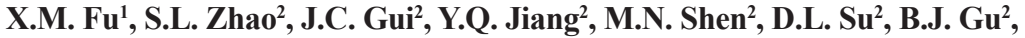 \\ X.Q. Wang' ${ }^{2}$ Q.J. Ren' ${ }^{2}$ X.D. Yin', W.B. Huang' ${ }^{2}$ and X.G. Chen ${ }^{2}$ \\ ${ }^{1}$ Department of Neurology, Nanjing Hospital of Traditional Chinese Medicine, \\ Nanjing, China \\ ${ }^{2}$ Department of Rheumatology, Nanjing First Hospital, \\ Nanjing Medical University, Nanjing, China \\ Corresponding author: X.G. Chen \\ E-mail: chexguo@163.com \\ Genet. Mol. Res. 14 (2): 4513-4520 (2015) \\ Received July 3, 2014 \\ Accepted December 10, 2014 \\ Published May 4, 2015 \\ DOI http://dx.doi.org/10.4238/2015.May.4.9
}

\begin{abstract}
Hemangioblastoma of the central nervous system occurs as sporadic tumors or as a part of von Hippel-Lindau (VHL) disease, an autosomal dominant hereditary tumor syndrome caused by a germline mutation in the $V H L$ tumor suppressor gene. We screened a Chinese family with VHL for mutations in the VHL gene and evaluated a genetic test for diagnosing VHL disease and clinical screening of family members. DNA extracted from the peripheral blood of all live members and from tissue of deceased family members with VHL disease was amplified by polymerase chain reaction to $3 \mathrm{VHL}$ gene exons. Mutations in the amplification products were compared against the Human Gene Mutation Database. The involvement of multiple organs among the kindred with VHL disease was confirmed by medical history and radiography. Of the 12 members of the 4-generation family, 5 were diagnosed with VHL disease. Patient age at the initial diagnosis was 26-36 years (mean $=31$ years). The mean time was 15 months
\end{abstract}


(11-19 months) from symptom appearance to the first patient visit to the hospital. Sequence analysis revealed that the frameshift mutation 327del C (p.Gly39Alafs*26) in exon 1 affected all family members, but not the healthy individuals or 16 unrelated controls. Members without gene mutation showed no clinical manifestation of VHL disease. We detected a conserved novel frameshift mutation in the VHL gene of the family members that contributes to VHL. DNA analysis of VHL is advantageous for VHL diagnosis. We developed a quick and reliable method for VHL diagnosis.

Key words: Central nervous system; Hemangioblastoma; VHL gene; Neuropathology; von Hippel-Lindau disease

\section{INTRODUCTION}

von Hippel-Lindau (VHL) disease is an autosomal dominant inherited cancer syndrome characterized by a predisposition to developing various neoplasms in multiple organs, including the central nervous system (CNS), retinal hemangioblastoma, renal cell carcinoma, pheochromocytoma, pancreatic tumor, endolymphatic sac tumor, and epididymal cystadenoma. The gene responsible for VHL disease is known as the VHL tumor suppressor gene (VHL gene), which was mapped at chromosome 3p25-26 (Latif et al., 1993). Hundreds of distinct germline mutations have been identified in European and Japanese families. Hemangioblastoma can be life-threatening if located at the spinal canal, and relapses have poor prognosis. The prevalence of VHL disease is estimated to be between 1 in 36,000 and 1 in 45,500. Retina angioma and CNS hemangioblastoma are the main clinical manifestations of the disease. CNS hemangioblastoma and renal cell carcinoma in patients with VHL disease are the 2 principal causes of death. Therefore, familial CNS hemangioblastoma is the main manifestation of VHL disease. Thus, early diagnosis is critical for this disease as well as for most hereditary cancer syndromes. VHL germline mutations are associated with hereditary hemangioblastoma of the CNS. Mutations in the VHL gene inhibit or modulate cellular functions of the wild-type VHL protein (pVHL). To detect mutations, all VHL gene exons and their flanking intronic sequences were amplified and directly sequenced. We identified a novel mutation in a family with VHL. This mutation expands the database on VHL gene mutations in VHL. This study is expected to facilitate future genetic investigations and prenatal diagnoses.

\section{MATERIAL AND METHODS}

\section{Patients}

Records of Nanjing First Hospital patients surgically treated for CNS hemangioblastoma from 1990 to 2011 were reviewed. Among these patients, 5 from the same family were affected by VHL disease; these patients had been diagnosed according to established criteria. One family member with VHL disease was then selected. We investigated all members of this family. Three members died from neurological diseases 10 years ago. The symptoms were 
similar to those of CNS hemangioblastoma. Any child in the family was considered to be at risk of inheriting the disease. In the family, all members volunteered for genetic analysis. These members were required to undergo examination, including magnetic resonance imaging (MRI) of the CNS, abdominal ultrasound, and computed tomography, to collect clinical information regarding intracranial and spinal diseases and to obtain detailed data from ophthalmological and visceral findings. This study was approved by the medical Ethics Committee of Nanjing Medical University.

\section{Molecular genetic analysis}

Blood samples from the volunteers were subjected to $V H L$ mutation analysis regardless of their clinical manifestation of VHL disease. Genomic DNA was extracted from peripheral blood and stored tissues using an EZ-10 Spin Column Genomic DNA Minipreps Kit (Bio Basic, Inc., Ontario, Canada). Six sets of primers (Table 1) were designed to cover all 3 exons (Sangon, Shanghai, China). Sequences were analyzed and compared using the Phred-PhrapConsed software (version 12.0; http://bozeman.mbt.washington.edu/consed/consed.html).

Table 1. Primer sequences for VHL gene amplification and loss of heterozygosity analysis.

\begin{tabular}{lll}
\hline Location & Primer name & Primer sequences (5'-3') \\
\hline VHL-exon 1 & 1.1 -forward & AGGTCGACTCGGAGCGC \\
& 1.1 -reverse & CGTCTTCTTCAGGGCCGTAC \\
& 1.2 -forward & AGGCAGGCGTCGAAGAGTAC \\
& 1.2 -reverse & ACGCGCGGACTGCGATTGC \\
VHL-exon 2 & 1.3 -forward & CAGGTCATCTTCTGCAATCGC \\
& 1.3 -reverse & CTTCAGACCGTGCTATCGGTC \\
VHL-exon 3 & 2.0 -forward & GTGGCTCTTTAACAACCTTTGC \\
& 2.0 -reverse & CCTGTACTTACCACAACAACCTTATC \\
& 3.1 -forward & TGTACTGAGACCCTAGTCTGC \\
& 3.1 -reverse & GCGCTCCTGTGTCAGCCGCTC \\
& 3.2 -forward & TGGAAGACCACCCAAATGTGC \\
\hline
\end{tabular}

\section{Cervical spine MRI}

All MRI scans were obtained using a 1.5T system (Eclipse Systems, Inc., Branford, CT, USA). The scans included sagittal $T 1$ (repetition time, $T R=500 \mathrm{~ms}$, echo time, $T E=12$ $\mathrm{ms})$, sagittal $T 2(T R=3000 \mathrm{~ms}, T E=112 \mathrm{~ms})$, sagittal short tau inversion recovery $(T R=2864$ $\mathrm{ms}, T E=1.4 \mathrm{~ms}, T I=150 \mathrm{~ms})$, and axial gradient-recalled $T 2(T R=500 \mathrm{~ms}, T E=18 \mathrm{~ms}$, flip angle $=40^{\circ}$ ) sequences with a scan thickness of $3.0 \mathrm{~mm}$ and intervals of $0.5 \mathrm{~mm}$.

\section{Hematoxylin and eosin $(\mathrm{H} \& \mathrm{E})$ staining}

The specimens were fixed in $10 \%$ buffered formalin and processed for paraffinembedded tissues. Sections with a thickness of $5 \mu \mathrm{m}$ were cut and stained with H\&E using an automatic dyeing machine (Sakura, Tokyo, Japan) according to the manufacturer protocol. H\&E-stained sections were examined by light microscopy (BX41, Olympus, Tokyo, Japan). 


\section{RESULTS}

\section{Clinical material}

The pedigrees of the family with VHL disease are shown in Figure 1. Five members of the Chinese family had VHL and a common manifestation of CNS hemangioblastoma. The patients initially diagnosed with VHL were aged 26-36 years (mean $=31$ years), and the mean time was 15 months (11-19 months) from the emergence of symptoms to the patients' first visit to the hospital. All patients had undergone operation, and excised tissues were stored in the Department of Pathology for H\&E staining.
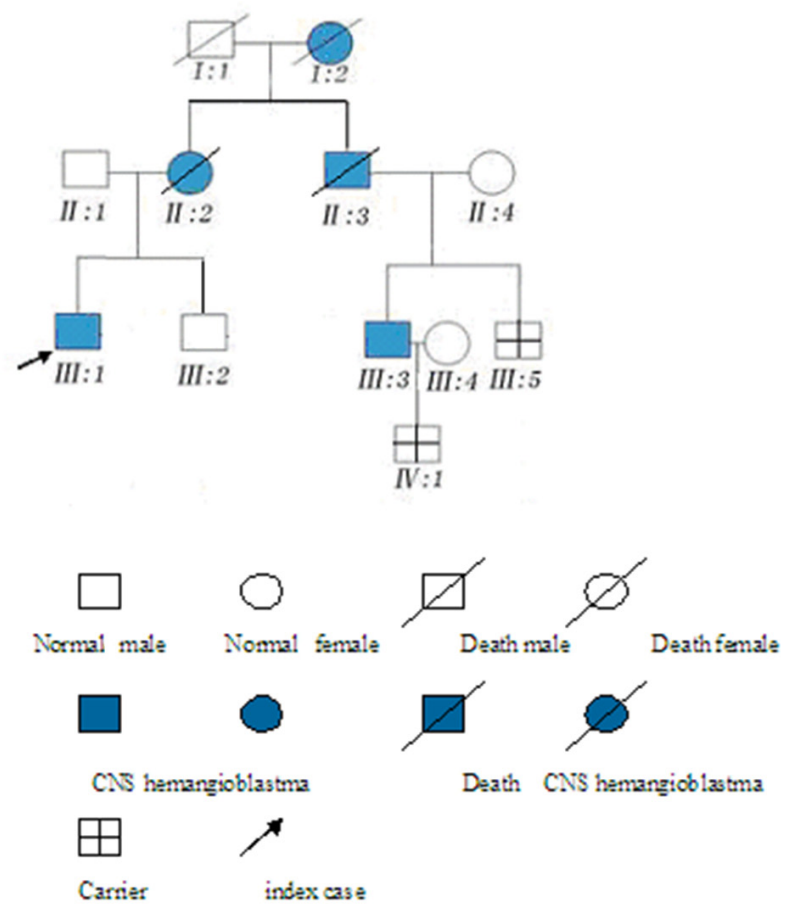

Figure 1. Pedigree depicting the phenotype in the Chinese kindred. VHL disease in the kindred was classified as type I. Most of the patients died of CNS hemangioblastoma. All members volunteered for genetic analysis. Two carriers with VHL gene mutation (III:5, IV:1), three deceased patients (I:2, II:2, II:3), and two symptomatic patients (III:1, III:3) were identified.

\section{Cervical spine MRI}

Two symptomatic patients (III:1, III:3) suffered from progressive severe neck pain and numbness of the left arm for 6 months. Both patients were initially treated with opioids and antiinflammatory medications and wore rigid neck collars. Despite intense conservative therapy, both patients experienced inadequate pain relief. Both patients underwent MRI scans preoperatively. A cervical spine MRI scan showed cystic-solid signal intensity with a large amount of liquid in the craniocervical conjunction on T1-weighted and T2-weighted (T2-W) images (Figure 2). 


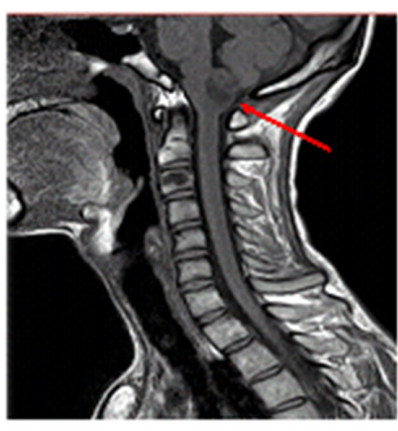

Tive

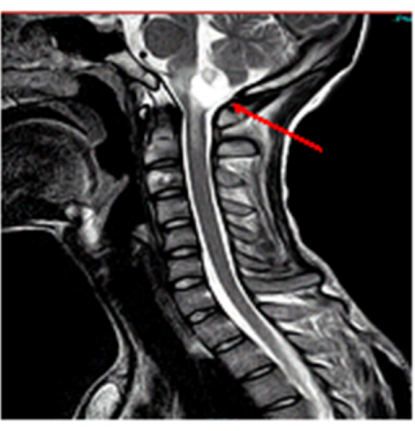

T2Vu

Figure 2. Cervical spine MRI scan. MRI findings suggestive of hemangioma in the $\mathrm{C} 3$ vertebra present a lesion of the long T1, T2 signal intensity, and high signal intensity in the STIR image. MRI findings suggestive of occupying change show cystic-solid signal intensity with a large amount of liquid in the craniocervical conjunction in T1weighted and $\mathrm{T} 2$-weighted images.

\section{H\&E staining}

Microscopic analysis revealed 2 components of the tumor. These components were vacuolated, lipid-rich stromal cells, and a fine capillary network. The ovoid to polygonal stromal cells contained small, hyperchromatic or pyknotic nuclei. Mitosis was not observed. Several capillaries were lined with hyperplastic endothelial cells, but several others were dilated with endothelial cells of normal appearance (Figure $3 a$ and $b$ ).

a

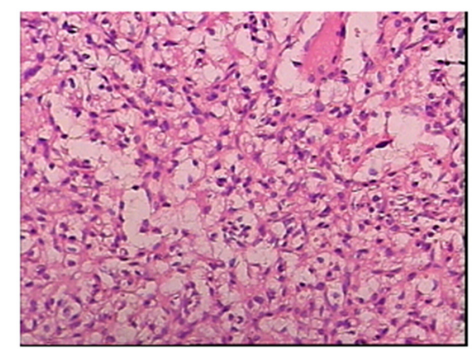

b

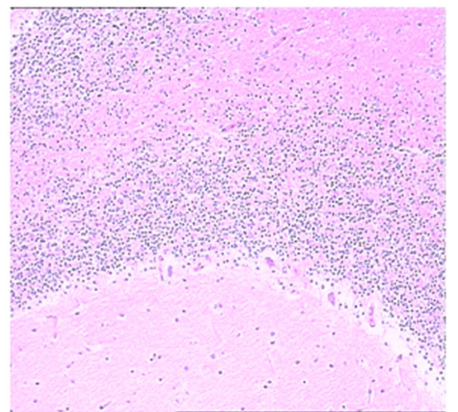

Figure 3. a. Histopathologic hallmark of the tumor composed of vacuolated, lipid-rich stromal cells and a fine capillary network. The stromal cells are ovoid to polygonal with vacuolated cytoplasm, and the vessels are dilated with hyperplastic endothelial cells. b. The cerebellum is intact and has a distinct pattern of Purkinje's cell layer, a granular layer, and a molecular layer. No inflammatory and tumor cells are observed in the cerebellum. 


\section{$V H L$ gene mutation}

Genomic DNA was extracted from peripheral blood and stored tissues. Six sets of primers were designed to cover all 3 exons. Sequences were analyzed and compared using the Phred-Phrap-Consed software. Polymerase chain reaction products were directly sequenced by the BGI Company (Shenzhen, China). Sequencing reactions were achieved with the same primers used to amplify exons. Sequences were analyzed using the BioEdit software. According to the human reference sequence NM_000551.3, a frameshift mutation 327 cytosine deletion was observed in exon 1 of the $V H L$ gene. This deletion changed the amino acid sequence from 39 glycine, creating a premature stop codon (GTG>TGA) between codons 65 and 66 (p.Gly39Alafs*26) and inhibiting VHL protein function (Figure 4). Healthy individuals and 16 unrelated controls were heterozygous for the detected change.

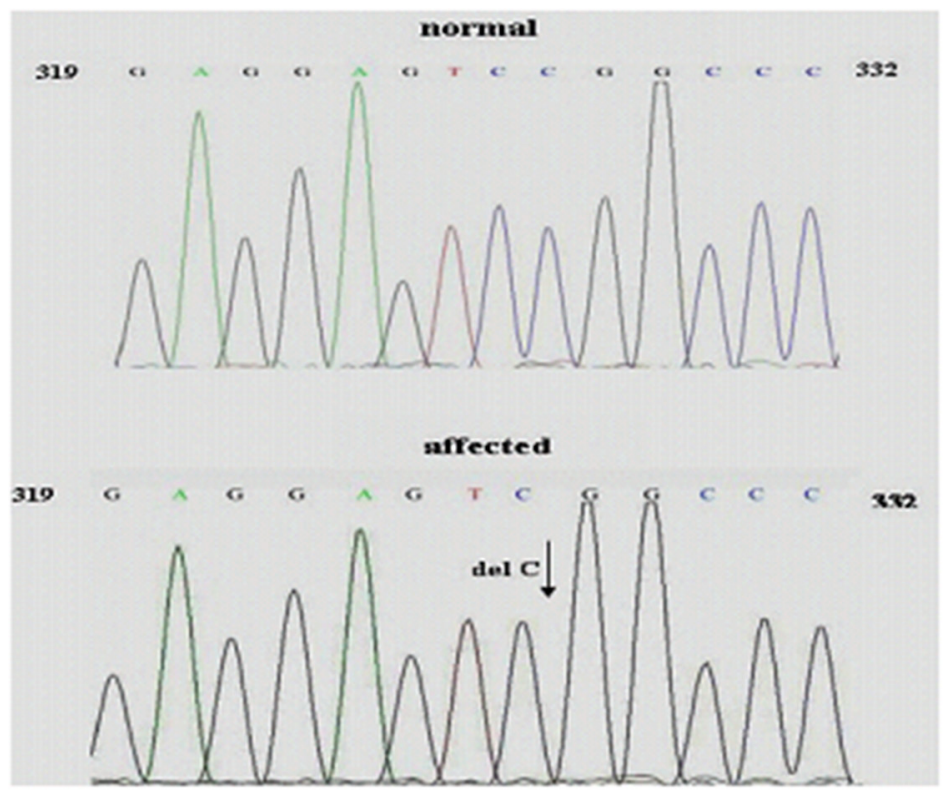

Figure 4. Analysis of VHL genes of a VHL-affected Chinese family. Frameshift mutation 327del C (p.Gly39Alafs*26) in exon 1 was found in all affected members but not in the healthy individuals or the 16 unrelated controls.

\section{DISCUSSION}

VHL disease is a hereditary neoplasm syndrome with complex manifestations, primarily CNS hemangioblastoma, renal cysts, pancreatic cysts, and retinal angioma. The most common cause of VHL-related death is CNS hemangioblastoma. The American National Cancer Institute has established a clinical classification system that categorizes families without pheochromocytoma as type I and those with pheochromocytoma as type II. The Chinese family contained no members with pheochromocytoma and was thus categorized as type I. 
Hemangioblastoma of the CNS occurs as a part of VHL disease. Early diagnosis remains a problem because the syndrome shows wide variations in complex manifestations. Moreover, pedigree is seldom analyzed. Previous studies have reported that $86-100 \%$ of familial hemangioblastoma patients contain VHL gene mutations (Stolle et al., 1998; Gläsker et al., 1999; Gijtenbeek et al., 2002; Jia et al., 2013), as hemangioblastoma of the CNS is the most common preceding manifestation of VHL disease. Thus, molecular genetic analysis of the VHL gene significantly affects VHL disease diagnosis.

The VHL gene contains 3 exons and encodes an mRNA of $4.5 \mathrm{~kb}$ with a long 3 '-untranslated region. The $V H L$ gene is a very important tumor suppressor gene. Inactivation of the VHL tumor suppressor gene is an early causal event in the development of familial CNS hemangioblastoma. The mutation phenotype of the VHL gene includes deletion, missense, nonsense, frameshift, and insertion mutations. Zbar et al. (1996) reported germline mutations in 469 VHL families from North America, Europe, and Japan. Common germline VHL mutations included delPhe76, Asn78Ser, Arg161Stop, Arg167Gln, Arg167Trp, and Leu178Pro. Approximately $15-20 \%$ of the mutations were deletion mutations, $27 \%$ were missense mutations, and $27 \%$ were nonsense mutations. We identified mutations in exon 1 and the first half of exon 3, which is consistent with the report of Zbar et al. (1996). The VHL gene product, VHL protein, has several functions, including downregulation of hypoxia-inducible mRNA, proper assembly of the extracellular fibronectin matrix, regulation of exit from the cell cycle, and expression regulation of carbonic anhydrases 9 and 12 (Kondo and Kaelin Jr., 2001).

In the present study, a frameshift mutation $(327 \mathrm{del} \mathrm{C})$ in exon 1 was detected in all affected members, but not in healthy individuals or in the 16 unrelated controls. This mutation resulted in changes at codon 39 from Gly to Ala in the Chinese family. We hypothesize that the germline $V H L$ mutation (p.Gly39Alafs*26) is an active mutation location.

Melmon and Rosen (1964) described diagnostic criteria for VHL disease. If a family has a history of CNS hemangioblastoma, only 1 hemangioblastoma or visceral lesion is required for a positive diagnosis of VHL disease. It is difficult to diagnose each affected patient using clinical criteria because VHL disease manifestations depend on age. Patients are typically diagnosed with CNS hemangioblastoma in their twenties. Stolle et al. (1998) detected germline mutations in the VHL gene in all VHL families tested. Enhanced detection methods will significantly increase the usefulness of $V H L$ genetic analysis on the clinical management of VHL disease.

The detected mutation is novel and has not been described previously. The clinical features of the third family were early morbidity, rapid progress, high relapse rate, and high mortality rate, indicating the effects of the detected mutation. Zhang et al. (2007) identified 9 mutations that differed from our study when they tested 9 Chinese families with VHL disease. Seven of the mutations were in exon 1, whereas the other 2 were in exon 2 . The test results indicate that Chinese VHL patients contain gene germline mutations, but whether these patients contain the mutation hotspot remains unknown. The relationship between tumor type and specific site of gene mutation requires confirmation by large-sample testing.

We identified a gene mutation in all VHL-affected family members who showed no lesions of the disease complex. Molecular genetic testing is the only method suitable for VHL diagnosis because a complete clinical screening of all family members would be excessive and result in low compliance (based on our experience). Such screening is also expensive and unsafe, particularly for young family members, who are unlikely to show manifestations. Thus, mutation carriers in the family should urgently be identified by molecular genetic analysis. In 
this study, genetic testing revealed 2 mutation carriers in individuals with no known manifestation of VHL disease, likely because of the young ages of the patients (all were younger than 22 years). A follow-up study of these subjects is thus recommended.

DNA analysis of $V H L$ germline mutations is accurate and more effective than other diagnostic methods. Because CNS hemangioblastoma is an early manifestation of VHL disease, all patients with this symptom should be screened for VHL disease germline mutations. Such screening provides key information for VHL to facilitate the management of VHL disease.

\section{Conflicts of interest}

The authors declare no conflict of interest.

\section{ACKNOWLEDGMENTS}

Research supported by NSFC (Joint Research Fund for Young Scholars in Hong Kong and Abroad; \#81228018), the Science and Technology Commission of Nanjing Municipality, and Key Programs of Medical Science and Technology Development Foundation of the Nanjing Department of Health (\#201108026, \#ZKX020).

\section{REFERENCES}

Gijtenbeek JM, Jacobs B, Sprenger SH, Eleveld MJ, et al. (2002). Analysis of von Hippel-Lindau mutations with comparative genomic hybridization in sporadic and hereditary hemangioblastomas: possible genetic heterogeneity. J. Neurosurg. 97: 977-982.

Gläsker S, Bender BU, Apel TW, Natt E, et al. (1999). The impact of molecular genetic analysis of the VHL gene in patients with hemangioblastomas of the central nervous system. J. Neurol. Neurosurg. Psychiat. 67: 758-762.

Jia D, Tang B, Shi Y, Wang J, et al. (2013). A deletion mutation of the VHL gene associated with a patient with sporadic von Hippel-Lindau disease. J. Clin. Neurosci. 20: 842-847.

Kondo K and Kaelin WG Jr (2001). The von Hippel-Lindau tumor suppressor gene. Exp. Cell. Res. 264: 117-125.

Latif F, Tory K, Gnarra J, Yao M, et al. (1993). Identification of the von Hippel-Lindau disease tumor suppressor gene. Science 260: 1317-1320.

Melmon KL and Rosen SW (1964). Lindau's disease. Review of the literature and study of a large kindred. Am. J. Med. 36: 595-617.

Stolle C, Glenn G, Zbar B, Humphrey JS, et al. (1998). Improved detection of germline mutations in the von HippelLindau disease tumor suppressor gene. Hum. Mutat. 12: 417-423.

Zbar B, Kishida T, Chen F, Schmidt L, et al. (1996). Germline mutation in the von Hippel-Lindau disease (VHL) gene in families from North American, Europe, and Japan. Hum. Mutat. 8: 348-357.

Zhang J, Huang YR, Pan JH, Liu DM, et al. (2007). Germline mutations in Chinese kindreds with von Hippel-Lindau syndrome. Chin. J. Med. Genet. 24: 124-127. 